\title{
Up/Down Impurity Density Asymmetries in C-Mod Plasmas
}

\author{
J.E. Rice, M.L. Reinke ${ }^{\dagger}$ N. Cao, J.W. Hughes, J.M.A. Ashbourn , D.R. Ernst, \\ A.E. Hubbard and J.H. Irby \\ PSFC, MIT, Cambridge, Massachusetts 02139, USA \\ ${ }^{\dagger}$ ORNL, Oak Ridge, Tennessee 37831, USA \\ ${ }^{b}$ Mathematical Institute, University of Oxford, Oxford OXI 3LB, UK
}

\begin{abstract}
Brightness profiles of $\mathrm{x}$-ray emission from $\mathrm{H}$-like $\mathrm{Ar}^{17+}$ exhibit a distinct up/down asymmetry under certain operating conditions in C-Mod plasmas, indicating that impurity densities are not constant on flux surfaces with $\mathrm{r} / \mathrm{a}$ between $\sim 0.8$ and $\sim 0.95$. In Land I-mode plasmas, there is an $\mathrm{x}$-ray brightness excess, up to a factor of 8 , on the side opposite to the ion $\mathbf{B} \times \nabla \mathbf{B}$ drift direction. This effect is not observed in H-mode plasmas, presumably due to edge impurity transport being dominated by a strong inward pinch, which is absent in L- and I-mode. The magnitude of the asymmetry in L- and I-mode decreases with increasing plasma current, similar to the observed decrease in radial impurity diffusivity. In I-mode, where the codependence between electron density and temperature can be broken with ICRF heating power, the asymmetry magnitude is found to decrease with increasing density and with increasing edge temperature at fixed density. These measurements exhibit some qualitative features of neo-classical expectations but the observed asymmetry magnitude is much larger than predicted and some scalings with plasma parameters are not seen. The up/down asymmetry appears to be largest when the cross field impurity diffusivity is the highest.
\end{abstract}




\section{Introduction}

The study and understanding of impurity behavior is important in fusion devices for a variety of reasons. Radiation losses from intrinsic impurities can lead to energy confinement degradation, which is particularly problematic in devices with high $\mathrm{Z}$ metallic walls in operational regimes such as $\mathrm{H}$-mode with near neo-classical particle transport. In addition, impurity seeding may be necessary for tailoring the radiation profile to avoid damage to plasma-facing components following heat exhaust, so care must be taken when injecting impurities. Impurity transport is sometimes used as a proxy for that of plasma ions since direct determination of particle transport coefficients is relatively difficult.

Radial impurity transport has been studied extensively (see Refs.[1-47] in [1]), often exhibiting anomalous characteristics, yet some aspects are consistent with neoclassical predictions. An interesting phenomenon in parallel impurity transport, which has been noted since the begining of tokamak operation, relates to the poloidal variation of impurity densities on flux surfaces. Recent work has focussed on inboard/outboard asymmetries $[2,3,4,5,6,7,8,9,10]$, again sometimes anomalous and sometimes in agreement with neo-classical theory. A longstanding curiosity is the up/down impurity density asymmetry, with an excess up to a factor of 10 opposite to the ion $\mathrm{B} \times \nabla \mathrm{B}$ drift direction $[11,12,13,14,15,16,17,18]$. The present study documents the parameter dependences of the magnitude of the up/down impurity asymmetry in various confinement regimes, expanding on the scalings for Ohmic plasmas [18]. Information on in/out impurity asymmetries is not available from the x-ray spectrometer system utilized here. After a brief description of the experimental set-up there is a presentation of $\mathrm{Ar}^{17+} \mathrm{Ly}_{\alpha} \mathrm{x}$-ray spectra and brightness profiles demonstrating the up/down asymmetry in section 2. Section 3 is dedicated to a description of the plasma parameter scalings of the magnitude of the asymmetry in L-, I- and H-mode plasmas, followed by a discussion in section 4 .

\section{Experimental Set-up}

The results described here were obtained from the Alcator C-Mod tokamak [19, 20], a compact $(\mathrm{R}=0.67 \mathrm{~m}, a \sim 0.21 \mathrm{~m}$, elongation $<1.8)$, high magnetic field $\left(\mathrm{B}_{\mathrm{T}}\right.$ $\leq 8 \mathrm{~T}$ ) device with molybdenum plasma-facing components. Auxiliary ICRF heating power [21] allows access to $\mathrm{H}$ - and I-mode [22, 23, 20, 24], in addition to Ohmic Lmode. For this study, plasma current and magnetic fields were in the range of $0.5 \mathrm{MA}$ $<\mathrm{I}_{\mathrm{P}}<1.3 \mathrm{MA}$ and 4.7 $\mathrm{T}<\mathrm{B}_{\mathrm{T}}<7.9 \mathrm{~T}$ for deuterium plasmas. Magnetic equilibrium was calculated using the EFIT code [25] from magnetics measurements. Electron temperature and density profiles were determined from Thomson scattering and ECE [26, 27], with $1.5 \mathrm{keV}<\mathrm{T}_{e}(0)<6.2 \mathrm{keV}$ and $0.6 \times 10^{20} / \mathrm{m}^{3}<\mathrm{n}_{e}<3.3 \times 10^{20} / \mathrm{m}^{3}$ in the cases described here. In the edge region, the electron and ion temperatures are typically the same $[28,29,30]$. X-ray spectra from $\mathrm{Ar}^{17+}$ were obtained from a spatially imaging high-resolution Johann spectrometer system with complete up/down coverage of the plasma cross-section [31,32]. All of the plasmas presented here were diverted 
with single null, in the 'reversed' magnetic field configuration with the ion $\mathrm{B} \times \nabla \mathrm{B}$ drift direction upwards. While toroidal rotation is important for in/out impurity asymmetries, it does not seem to be a factor for the up/down asymmetry. As will be seen in section 3, there is a big difference in the up/down asymmetry between I- and H-mode, while the toroidal rotation (with Mach numbers as large as 0.3) in these two regimes is quite similar [33]. Conversely, the rotation in L- and I-mode is very different [17], while the up/down asymmetry is similar.

Argon was introduced into the plasma through a piezo-electric valve, with core concentrations typically between $10^{-3} \mathrm{n}_{e}$ and $10^{-4} \mathrm{n}_{e}$. A representative spectrum of the $\mathrm{Ar}^{17+} \mathrm{Ly}_{\alpha}$ doublet and satellites [34, 35], taken through a central viewing chord, for a 5.4 T, 0.8 MA deuterium Ohmic L-mode discharge with $\left\langle\mathrm{n}_{e}>=0.8 \times 10^{20} / \mathrm{m}^{3}\right.$ and $\mathrm{T}_{e}(0)=3 \mathrm{keV}$, is shown in Fig.1. Population of the upper levels of the doublet

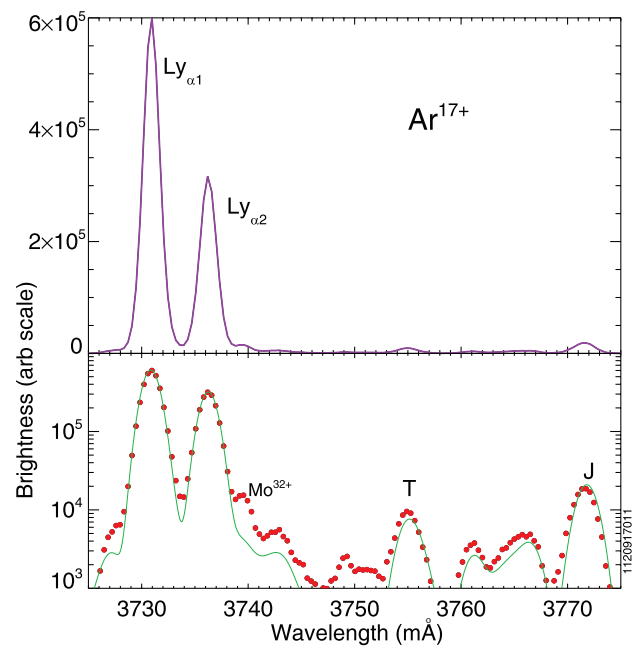

Figure 1: X-ray spectrum of the $\mathrm{Ar}^{17+} \mathrm{Ly}_{\alpha}$ doublet and satellites for a 5.4 T, 0.8 MA deuterium Ohmic L-mode discharge with $\left\langle\mathrm{n}_{e}\right\rangle=0.8 \times 10^{20} / \mathrm{m}^{3}$ and $\mathrm{T}_{e}(0)=3 \mathrm{keV}$. In the bottom frame a synthetic spectrum is shown for comparison by the solid green line.

is through collisional excitation of $\mathrm{H}$-like $\mathrm{Ar}^{17+}$ and radiative recombination of fully stripped $\mathrm{Ar}^{18+}$ (and through cascades from higher $\mathrm{n}$ levels). The rate coefficients for 
these processes depend upon the electron temperature, and the line brightness is proportional to the argon density for $\mathrm{n}_{e}$ and $\mathrm{T}_{e}$ fixed on a flux surface. Collisional-radiative modeling of the doublet is available from the COLRAD code $[36,37,38]$ and satellite intensity factors can be found in Ref.[39]. A synthetic spectrum [35] is shown in the bottom frame for comparison. The imaging $\mathrm{x}$-ray spectrometer system can view the entire plasma cross-section, and a comparison of the $\mathrm{Ar}^{17+} \mathrm{Ly}_{\alpha}$ doublet from viewing chords tangent to $\mathrm{r} / \mathrm{a}=0.8$ from the top and bottom of the same plasma as in Fig. 1 is shown in Fig. 2. The brightness from the bottom of the plasma is about a factor of 3

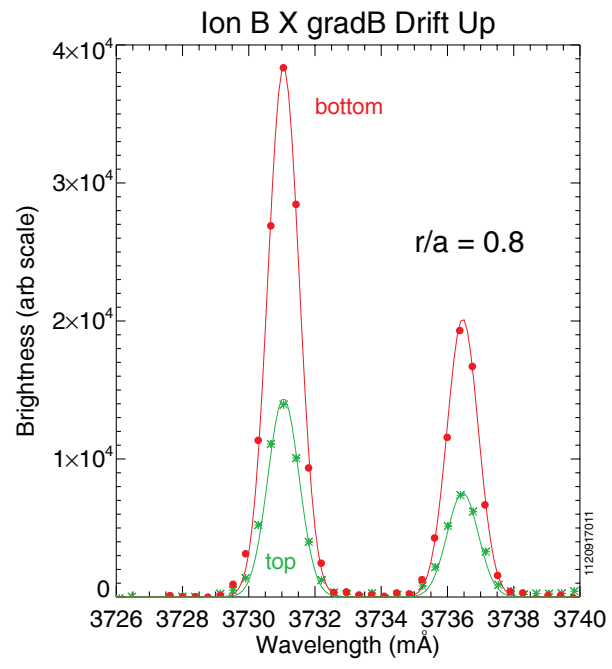

Figure 2: Spectra of the $\mathrm{Ar}^{17+} \mathrm{Ly}_{\alpha}$ doublet from viewing chords tangent to $\mathrm{r} / \mathrm{a}=0.8$, from the bottom (red dots and solid line) and top (green asterisks and chain line) of the plasma of Fig. 1.

larger than that from the top. This excess on the side opposite to the ion $\mathrm{B} \times \nabla \mathrm{B}$ drift direction has been previously noted $[16,18,17]$. Complete top and bottom $\mathrm{Ar}^{17+} \mathrm{Ly}_{\alpha 1}$ brightness profiles from a $5.4 \mathrm{~T}, 1.1 \mathrm{MA}\left(\mathrm{q}_{95} \sim 3.1\right)$ I-mode discharge, with $\left\langle\mathrm{n}_{e}\right\rangle=$ $1.3 \times 10^{20} / \mathrm{m}^{3}$ and $\mathrm{T}_{e}(0)=4.4 \mathrm{keV}$, are shown in Fig. 3. (I-mode features H-mode like energy confinement with L-mode like particle transport [23, 24].) The profiles are up/down symmetric inside $\mathrm{r} / \mathrm{a}=0.5$, while there is a distinct excess of over a factor of 2 on the side opposite to the ion $\mathrm{B} \times \nabla \mathrm{B}$ drift direction, which peaks near $\mathrm{r} / \mathrm{a} \sim 0.85$. 


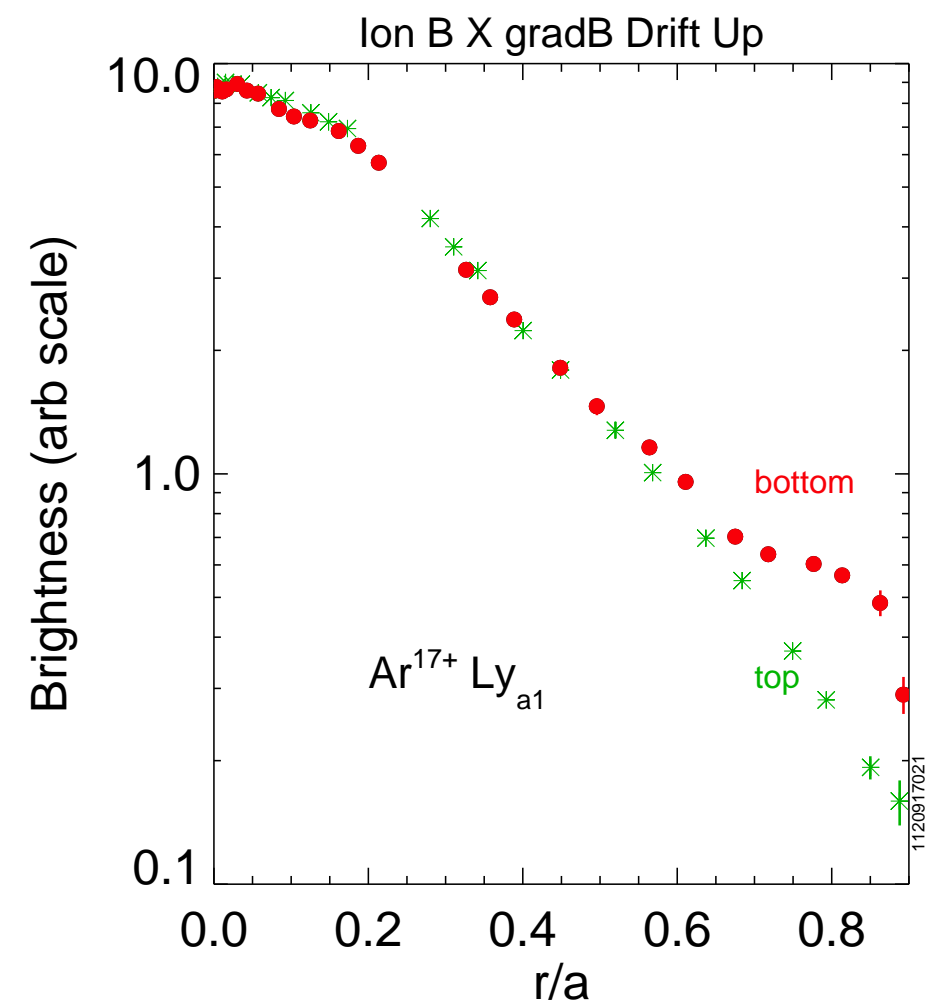

Figure 3: $\mathrm{Ar}^{17+} \mathrm{Ly}_{\alpha 1}$ brightness profiles from the bottom (red dots) and top (green asterisks) of a 5.4 T, 1.1 MA deuterium I-mode plasma. 
Outside $\mathrm{r} / \mathrm{a}=0.9$ there is very little $\mathrm{x}$-ray signal and the uncertainties are large. These brightness profiles have been inverted to find the $\mathrm{Ar}^{17+} \mathrm{Ly}_{\alpha 1}$ emissivity profile $[7,18]$, by separating the emissivity

$$
\epsilon=\epsilon_{0}(\psi)+\epsilon_{1}(\psi) \sin (\theta)
$$

into a symmetric $\mathrm{m}=0$ component and an $\mathrm{m}=1$ component multiplied by the sine of the poloidal angle. The ratio of the $m=1$ to the $m=0$ components for this discharge is shown in Fig. 4. (Here the - sign indicates that the bottom emissivity is greater than the top

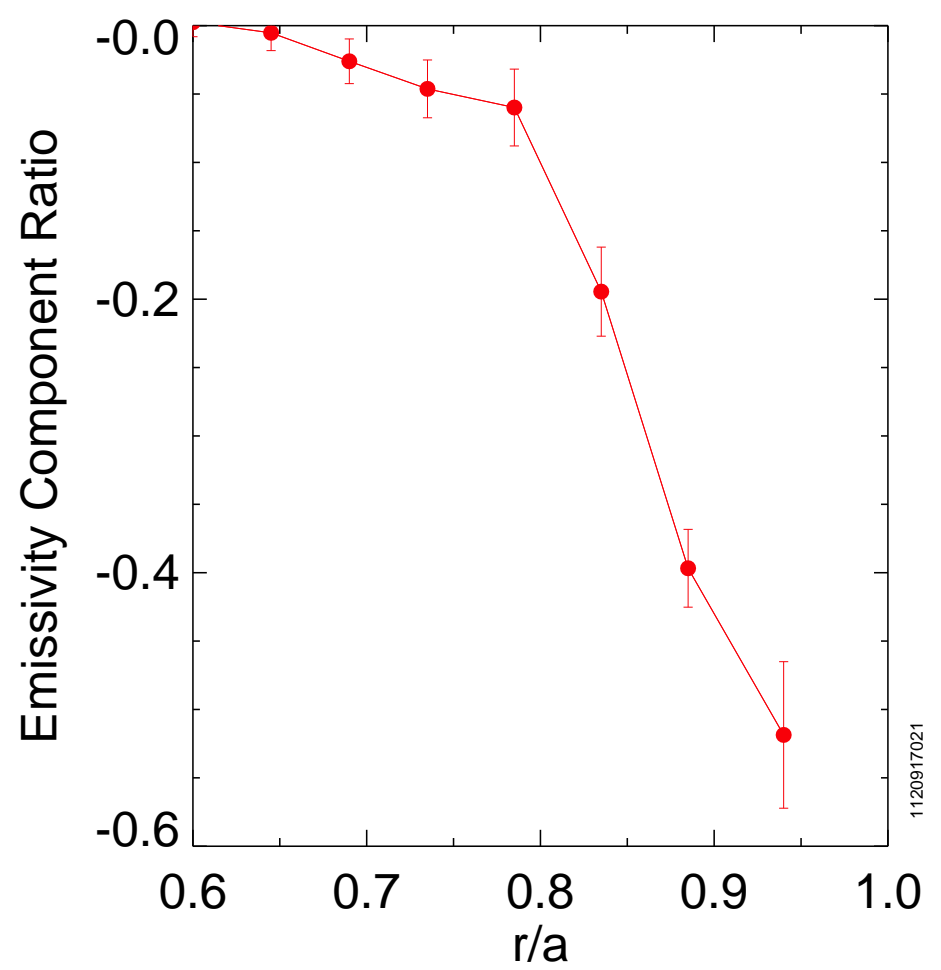

Figure 4: The ratio of the $\mathrm{m}=1$ component of the $\mathrm{Ar}^{17+} \mathrm{Ly}_{\alpha 1}$ emissivity profile to the $\mathrm{m}=0$ component for the 5.4 T, 1.1 MA deuterium I-mode plasma of Fig. 3.

in this 'reversed' magnetic field configuration.) The down/up asymmetry is very strong between $0.8<\mathrm{r} / \mathrm{a}<0.9$ [18].

For reference, complete electron temperature and density profiles for this discharge are shown in Fig.5. At r/a $\sim 0.85$, near the maximum of the up/down impurity asymmetry, the electron temperature was $\sim 1000 \mathrm{eV}$ with an electron density of $\sim 1.1 \times$ $10^{20} / \mathrm{m}^{3}$, for this discharge. The electron temperature gradient at this location was $\sim$ $25 \mathrm{keV} / \mathrm{m}$ with an electron density gradient of $\sim 5.6 \times 10^{20} / \mathrm{m}^{4}$. For this $\mathrm{q}_{95}=3.1$ 


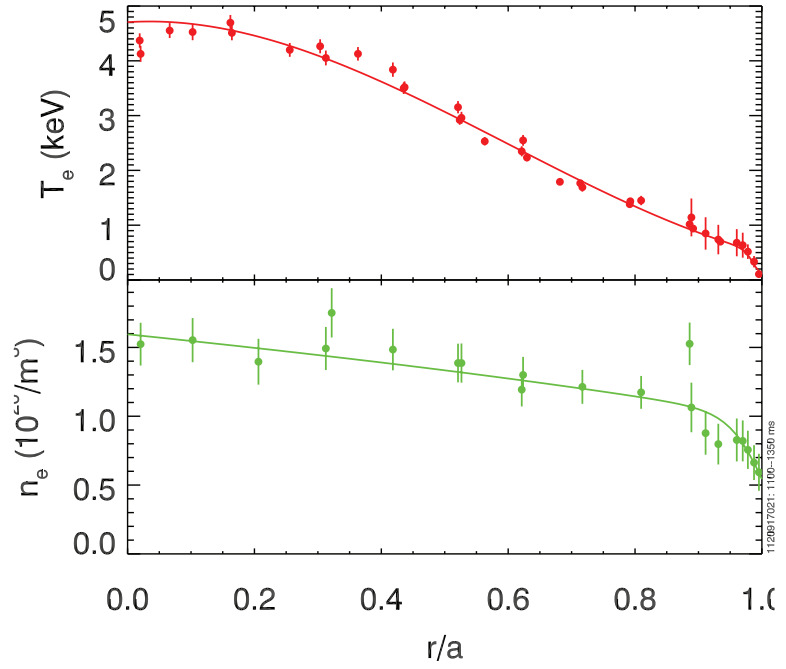

Figure 5: Radial profiles of the electron temperature (top, dots: Thomson scattering and ECE; line: analytic fit) and density (bottom, dots: Thomson scattering; line: analytic fit) for the 5.4 T, 1.1 MA deuterium I-mode plasma of Fig.3. 


\begin{tabular}{l|lccccccc}
\hline \hline Shot & Reg. & Ratio & $\mathrm{I}_{\mathrm{P}}$ & $\mathrm{n}_{e}$ & $\mathrm{~T}_{e}(0)$ & $\mathrm{T}_{e}(0.85)$ & $\mathrm{q}(0.85)$ & $\nabla \mathrm{n}_{e}(0.85)$ \\
\hline 1120917021 & $\mathrm{I}$ & 2.6 & 1.1 & 1.3 & 4.6 & 1.0 & 2.0 & $5.6 \pm 1.5$ \\
1120912027 & $\mathrm{O}$ & 6.6 & 0.6 & 0.8 & 2.4 & 0.3 & 3.5 & $1.4 \pm 1.4$ \\
1120829021 & $\mathrm{O}$ & 2.4 & 1.1 & 0.8 & 3.0 & 0.7 & 2.2 & $2.8 \pm 1.4$ \\
1121001018 & $\mathrm{I}$ & 3.3 & 0.8 & 1.3 & 2.9 & 0.6 & 2.6 & $3.8 \pm 1.7$ \\
1120913018 & $\mathrm{I}$ & 2.2 & 1.2 & 1.3 & 3.1 & 0.9 & 2.2 & $3.3 \pm 1.2$ \\
1120913010 & $\mathrm{I}$ & 2.3 & 1.0 & 1.7 & 3.8 & 0.6 & 2.2 & $8.3 \pm 1.7$ \\
1120906020 & $\mathrm{I}$ & 4.0 & 1.0 & 1.0 & 4.2 & 0.9 & 2.2 & $5.3 \pm 0.5$ \\
1120907005 & $\mathrm{~L}$ & 2.1 & 1.1 & 1.4 & 2.7 & 0.6 & 2.4 & $4.5 \pm 1.7$ \\
1120907005 & $\mathrm{H}$ & 1.0 & 1.1 & 2.6 & 3.5 & 0.9 & 2.4 & $-5.5 \pm 6.7$ \\
\hline
\end{tabular}

Table 1: Various plasma parameter values regarding the up/down impurity density asymmetry. First column: shot number; second column: confinement regime ( $\mathrm{O}$ for Ohmic L-mode, L for ICRF-heated L-mode, I for I-mode, H for H-mode); third column: down/up x-ray brightness ratio at $\mathrm{r} / \mathrm{a}=0.85$; fourth column: plasma current (MA); fifth column: average electron density $\left(10^{20} / \mathrm{m}^{3}\right)$; sixth column: central electron temperature $(\mathrm{keV})$; seventh column: electron temperature at $\mathrm{r} / \mathrm{a}=0.85(\mathrm{keV})$; eighth column: $\mathrm{q}$ at $\mathrm{r} / \mathrm{a}=0.85$; ninth column: edge electron density gradient at $\mathrm{r} / \mathrm{a}=0.85$ $\left(-10^{20} / \mathrm{m}^{4}\right)$.

plasma, the safety factor $\mathrm{q}$ value at $\mathrm{r} / \mathrm{a}=0.85$ was $\sim 2.0$. These and other parameters are recorded in the first row of Table 1 . There is no evidence in any C-Mod discharge for an up/down asymmetry in either the electron density or temperature, inside the last closed flux surface. In fact the parallel equilibration time for electrons is extremely fast, so none would be expected. (There is some evidence of an up/down asymmetry in the ion temperature profile and this will be discussed in a forthcoming publication.) In the following, since any asymmetry in the x-ray line emissivity can only be due to an asymmetry in the density of argon, the up/down brightness asymmetry will be used as a proxy for the up/down argon density asymmetry.

\section{Observed Scalings of the Asymmetry Magnitude with Plasma Parameters}

In this section, the scalings of the magnitude of the up/down impurity density asymmetry are explored. For simplicity, Ohmic L-mode discharges are examined first. The dependence of the asymmetry on density in Ohmic plasmas has been previously reported [17, 18], and showed a distinct turn on at fixed collisionality. A comparison of the up/down $\mathrm{Ar}^{17+} \mathrm{Ly}_{\alpha 1} \mathrm{x}$-ray brightness profiles for discharges at fixed electron density for two plasma currents, $0.6 \mathrm{MA}\left(\mathrm{q}_{95}=5.57\right)$ and $1.1 \mathrm{MA}\left(\mathrm{q}_{95}=3.48\right)$ is shown in Fig. 6. The asymmetry magnitude at $\mathrm{r} / \mathrm{a}=0.85$ for the $0.6 \mathrm{MA}$ discharge was 6.6, much larger than for the 1.1 MA plasma with a ratio of 2.4. Although the lower current plasma had a much narrower overall brightness profile due to the thinner electron temperature profile, the asymmetry was maximum near $\mathrm{r} / \mathrm{a} \sim 0.85$. Values for $\mathrm{q}(0.85)$ 


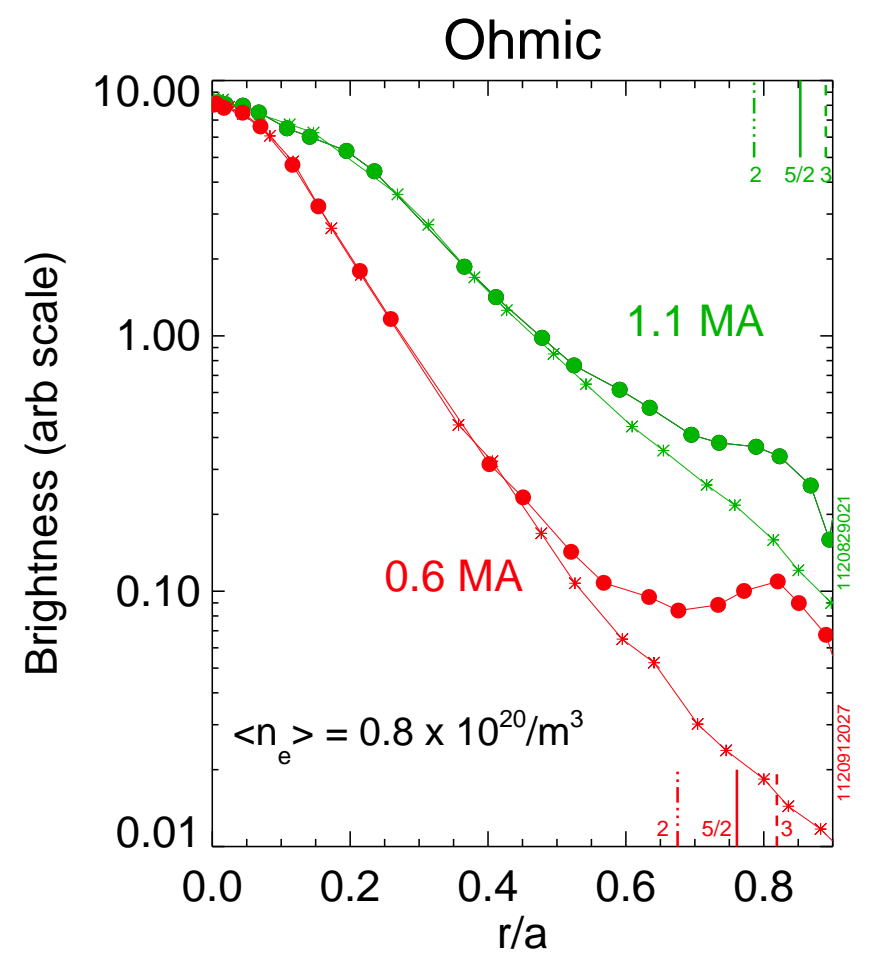

Figure 6: Bottom (dots) and top (asterisks) $\mathrm{Ar}^{17+} \mathrm{Ly}_{\alpha 1} \mathrm{x}$-ray brightness profiles for Ohmic L-mode discharges with $<\mathrm{n}_{e}>=0.8 \times 10^{20} / \mathrm{m}^{3}$ for plasma currents of $0.6 \mathrm{MA}$ (red) and 1.1 MA (green). $\mathrm{q}=2,5 / 2$ and 3 surface locations are indicated by the chain, solid and dashed lines, respectively, at the bottom in red for the 0.6 MA plasma and at the top in green for the 1.1 MA discharge. 
in the two cases were 3.5 and 2.2, respectively. Clearly there is no relation between the position of the maximum in the asymmetry and the local q value. These and other parameters are noted in second and third rows of Table 1. The up/down asymmetry has been examined for a range of plasma currents at fixed electron density for Ohmic L-mode discharges, and the results are presented in Fig. 7. There is a clear decrease of

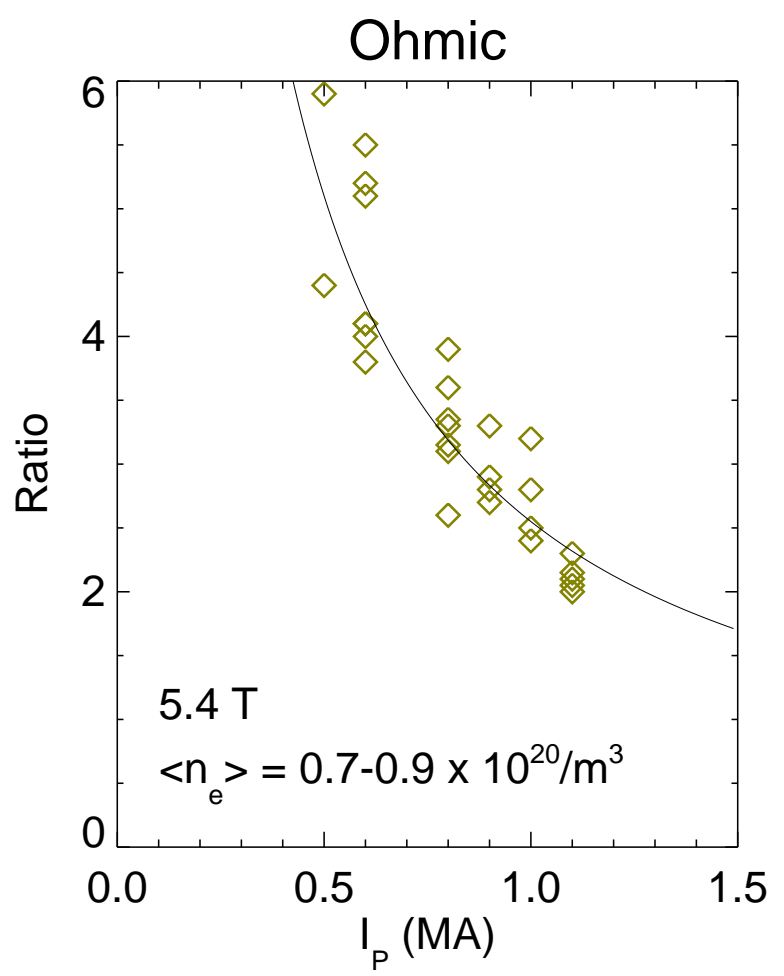

Figure 7: The down/up $\mathrm{Ar}^{17+} \mathrm{Ly}_{\alpha 1} \mathrm{x}$-ray brightness ratio at $\mathrm{r} / \mathrm{a}=0.8$ as a function of plasma current for Ohmic L-mode discharges with electron density between 0.7 and $0.9 \times 10^{20} / \mathrm{m}^{3}$. The solid line is proportional to $1 / \mathrm{I}_{\mathrm{P}}$.

the asymmetry with increasing plasma current, which is nearly inversely proportional. Here (and in the following) the brightness asymmetry at $\mathrm{r} / \mathrm{a}=0.80$ was used rather than $\mathrm{r} / \mathrm{a}=0.85$, since for this large number of discharges, some with relatively low argon levels, the scatter was smaller at $\mathrm{r} / \mathrm{a}=0.80$. This doesn't affect the general trends however. The plasmas listed in Table 1 are 'trophy shots' with very good signal levels at $\mathrm{r} / \mathrm{a}=0.85$.

I-mode plasmas yield similar observations. A comparison of up/down x-ray brightness profiles for two different plasma currents in I-mode discharges, formed with 3 MW of ICRF heating power, at an electron density of $\left\langle\mathrm{n}_{e}\right\rangle=1.3 \times 10^{20} / \mathrm{m}^{3}$ is shown in Fig. 8. Again, the lower plasma current discharge had a larger asymmetry. Param- 


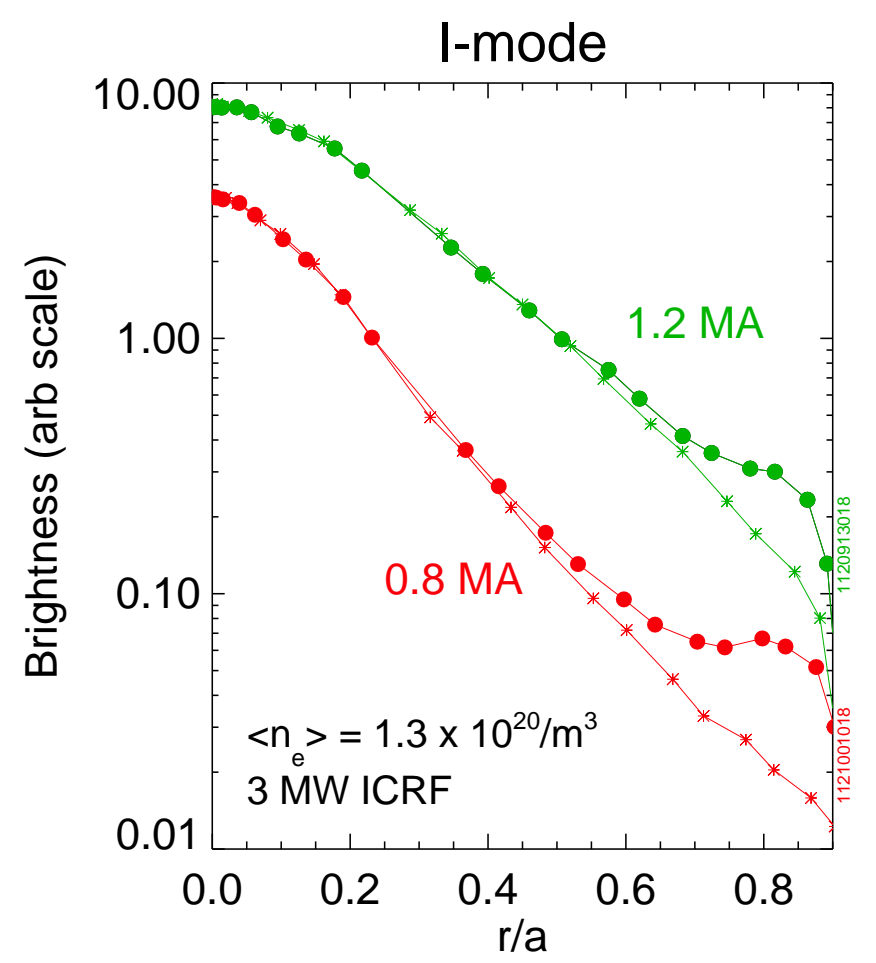

Figure 8: Bottom (dots) and top (asterisks) $\mathrm{Ar}^{17+} \mathrm{Ly}_{\alpha 1} \mathrm{x}$-ray brightness profiles for I-mode discharges with $\left\langle\mathrm{n}_{e}\right\rangle=1.3 \times 10^{20} / \mathrm{m}^{3}$ for plasma currents of $0.8 \mathrm{MA}$ (red) and 1.2 MA (green), both with $3 \mathrm{MW}$ of ICRF heating power. 
eters for these discharges are found in fourth and fifth rows of Table 1. The results of a complete plasma current scan at fixed density $\left(1.2\right.$ to $\left.1.4 \times 10^{20} / \mathrm{m}^{3}\right)$ for I-mode discharges is shown in Fig. 9. The decrease with increasing plasma current is apparent.

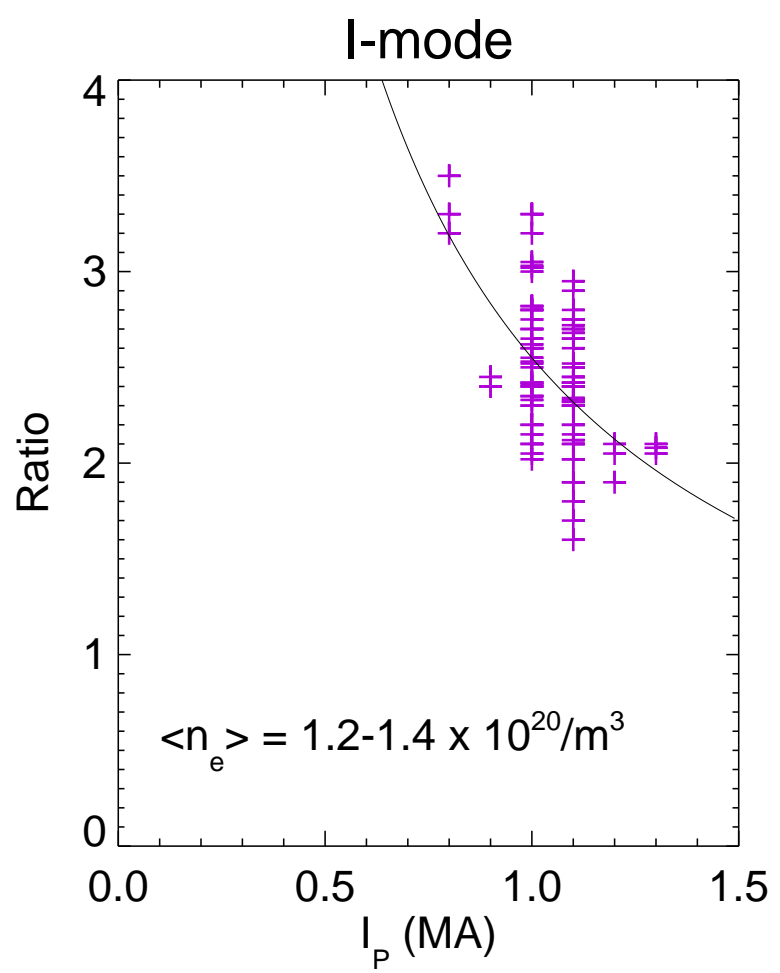

Figure 9: The down/up $\mathrm{Ar}^{17+} \mathrm{Ly}_{\alpha 1} \mathrm{x}$-ray brightness ratio at $\mathrm{r} / \mathrm{a}=0.8$ as a function of plasma current for I-mode discharges with electron density between 1.2 and $1.4 \times$ $10^{20} / \mathrm{m}^{3}$. The solid line is proportional to $1 / \mathrm{I}_{\mathrm{P}}$.

It should be noted that at fixed plasma current and electron density there is a large degree of scatter, due to a significant range of ICRF heating power and resulting electron temperature. In contrast, in Ohmic discharges there is a well-known one to one correspondence between electron density and temperature (see for example Figs. 17-19 in [17]). The effect of an increase in heating power/electron temperature is demonstrated in Fig. 10 which shows the time histories of an I-mode discharge with an increase in ICRF heating power. Between 1.0 and $1.05 \mathrm{~s}$ the power was increased from 1.6 to $4.2 \mathrm{MW}$. The central and edge electron temperature subsequently increased and the down/up x-ray brightness ratio decreased from $\sim 2.7$ to $\sim 1.6$. This relationship is summarized in Fig. 11 which shows the bottom/top $\mathrm{x}$-ray brightness ratio at $\mathrm{r} / \mathrm{a}=0.8$ as a function of the electron temperature at $\mathrm{r} / \mathrm{a}=0.8$ for $1.1 \mathrm{MA}$ I-mode discharges at fixed electron density $\left(1.2-1.4 \times 10^{20} / \mathrm{m}^{3}\right)$ for the full range of ICRF heating power. The 


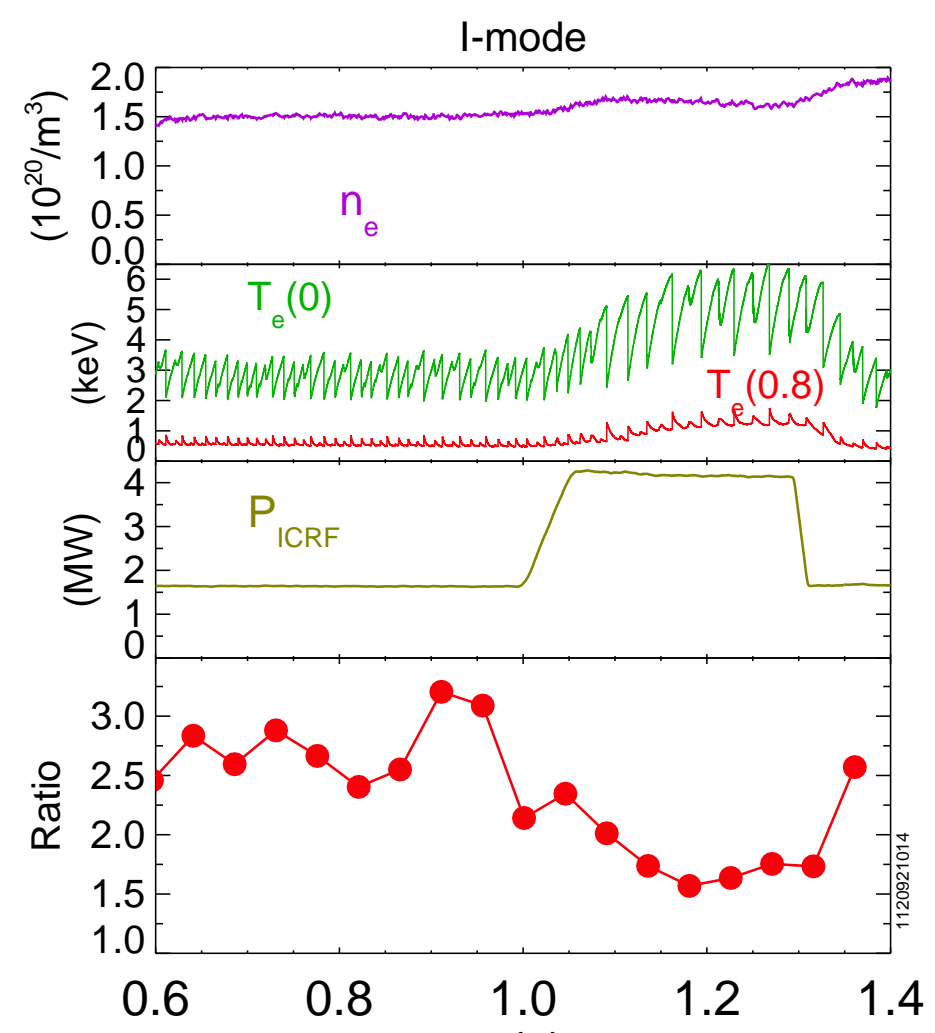

Figure 10: Time histories for a 1.2 MA ( $\left.\mathrm{q}_{95}^{\mathrm{t}}(\mathrm{S})_{3.0}\right)$ I-mode discharge. From top to bottom, the average electron density, the core and edge electron temperature, the ICRF heating power and the bottom/top $\mathrm{x}$-ray brightness ratio at $\mathrm{r} / \mathrm{a}=0.8$. 


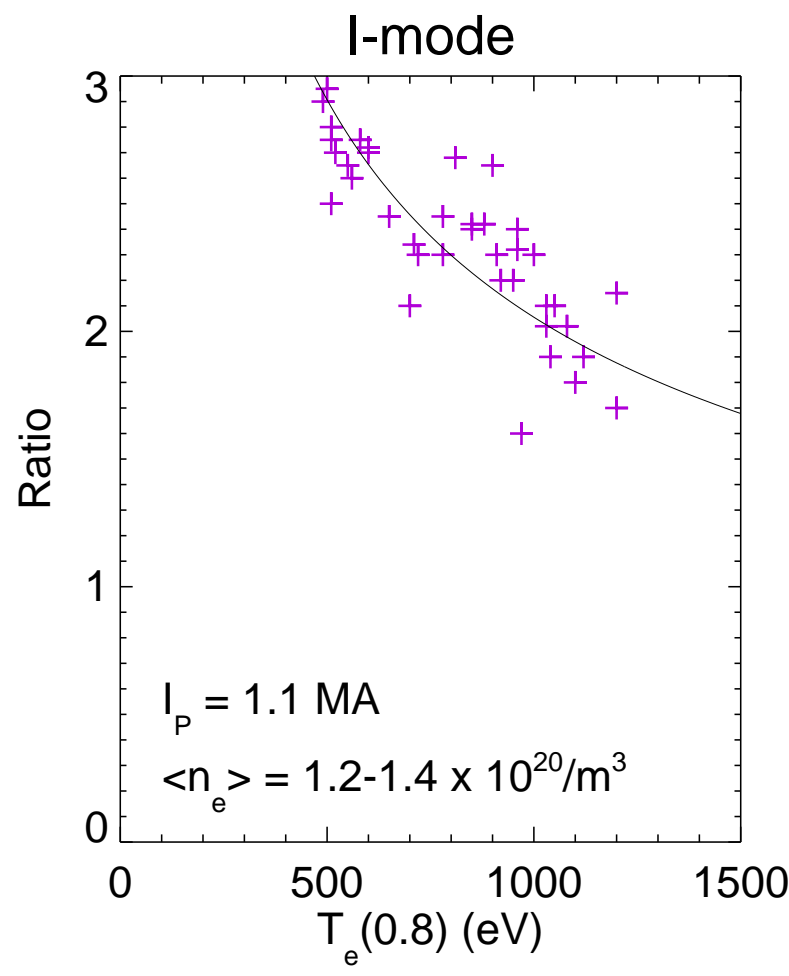

Figure 11: The down/up $\mathrm{Ar}^{17+} \mathrm{Ly}_{\alpha 1} \mathrm{X}$-ray brightness ratio at $\mathrm{r} / \mathrm{a}=0.8$ as a function of the electron temperature at $\mathrm{r} / \mathrm{a}=0.8$ for 1.1 MA I-mode discharges with electron density between 1.2 and $1.4 \times 10^{20} / \mathrm{m}^{3}$. The solid line is proportional to $1 / \sqrt{T_{e}}$. 
decrease with temperature scales approximately as $1 / \sqrt{T_{e}}$.

The previous examples were all demonstrated at fixed electron density. The magnitude of the asymmetry in I-mode depends also on the density, which is shown in Fig. 12, a comparison of the brightness profiles from 1.0 MA I-mode discharges with electron densities of 1.0 and $1.7 \times 10^{20} / \mathrm{m}^{3}$ (see the sixth and seventh rows of Table 1) at fixed ICRF heating power. The lower density discharge has a larger asymmetry.

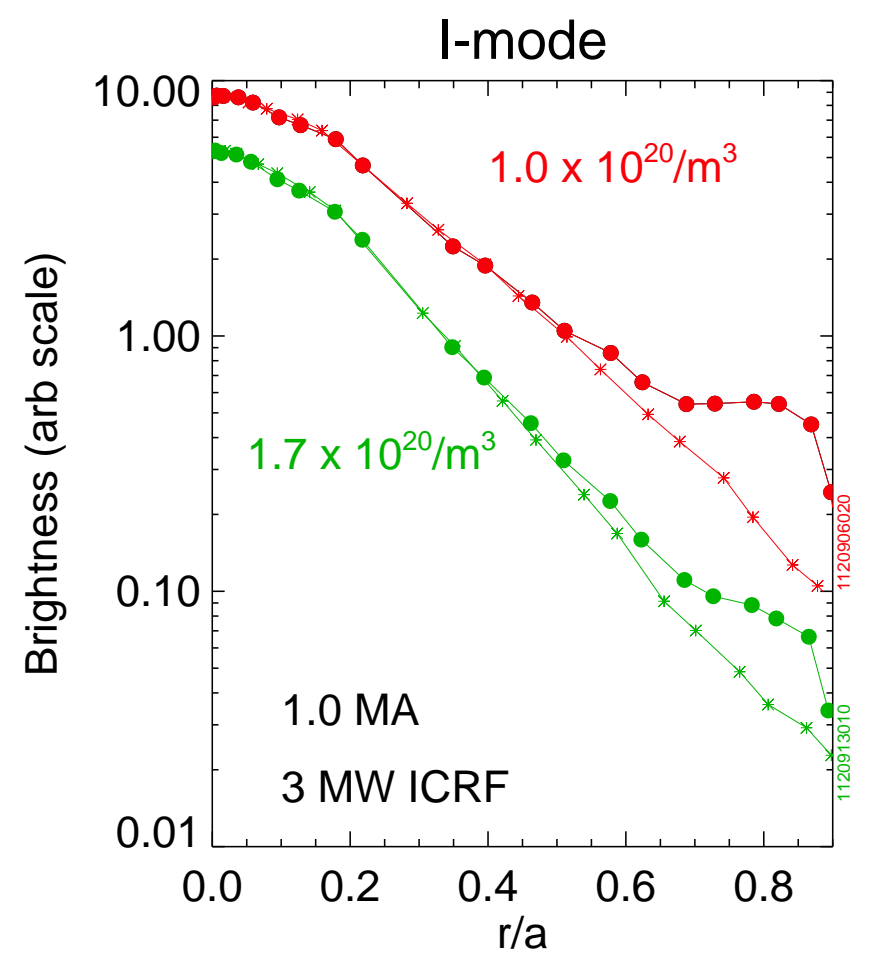

Figure 12: Bottom (dots) and top (asterisks) $\mathrm{Ar}^{17+} \mathrm{Ly}_{\alpha 1} \mathrm{x}$-ray brightness profiles for 1.0 MA I-mode discharges with electron densities of $1.0 \times 10^{20} / \mathrm{m}^{3}$ (red) and $1.7 \times$ $10^{20} / \mathrm{m}^{3}$ (green), both with $3 \mathrm{MW}$ of ICRF heating power.

The results of a complete scan with electron density at a fixed current of 1.0 MA and fixed ICRF power for I-mode plasmas are shown in Fig. 13. The x-ray brightness ratio decreases approximately as $1 / \mathrm{n}_{e}$.

The appearance of this up/down asymmetry pervades the L- and I-mode operational ranges but the asymmetry disappears during ELM-free H-mode. This is demonstrated in Fig. 14 which shows the down/up ratio becomes unity during the H-mode phase (0.79 $-0.99 \mathrm{~s}$ ) of the discharge. A distinguishing feature between either L- or I-mode and $\mathrm{H}$-mode is the pedestal density gradient (third panel). Radial brightness profiles from two different time slices for this discharge are shown in Fig.15. (Note that the H- 


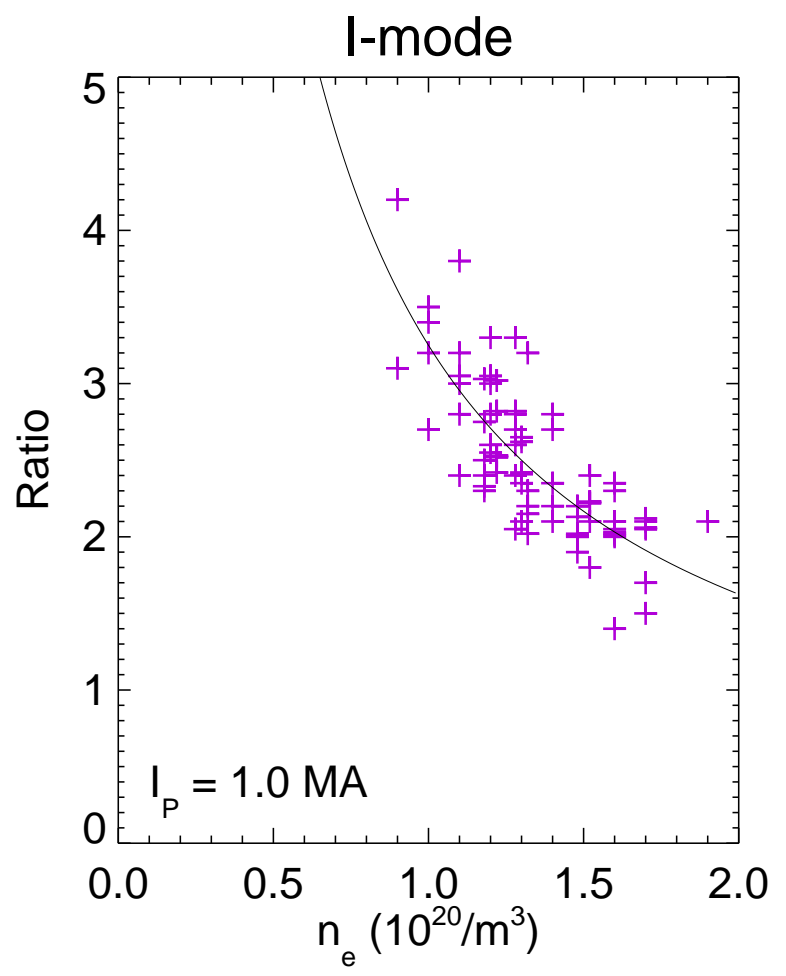

Figure 13: The down/up $\mathrm{Ar}^{17+} \mathrm{Ly}_{\alpha 1} \mathrm{x}$-ray brightness ratio at $\mathrm{r} / \mathrm{a}=0.8$ as a function of the electron density for 1.0 MA I-mode discharges. The solid line is proportional to $1 / \mathrm{n}_{e}$. 


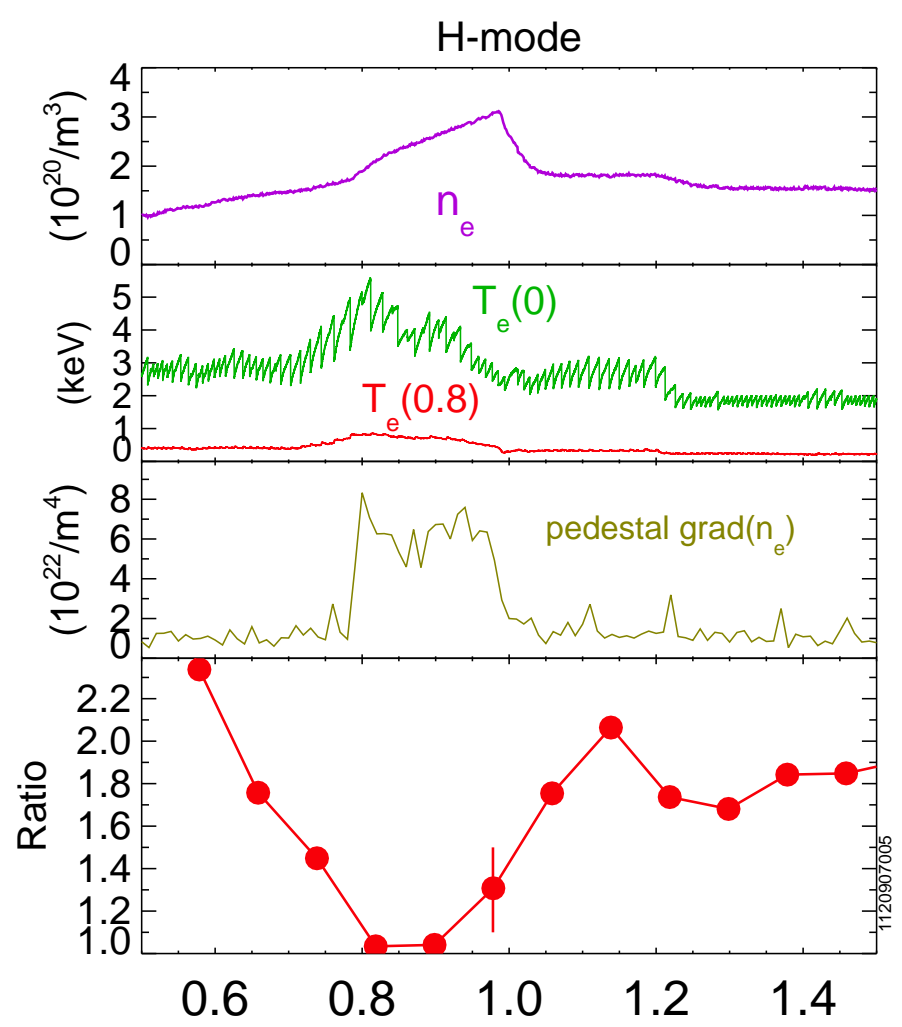

Figure 14: Time histories for a 1.1 MA ( $\left.\mathrm{q}_{55}^{\mathrm{t}}(\mathrm{S})_{3.7}\right)$ ICRF-heated L-mode discharge with an H-mode phase between 0.79 and $0.99 \mathrm{~s}$. From top to bottom, the average electron density, core and edge electron temperature, pedestal density gradient and bottom/top x-ray brightness ratio at r/a $=0.8$. 


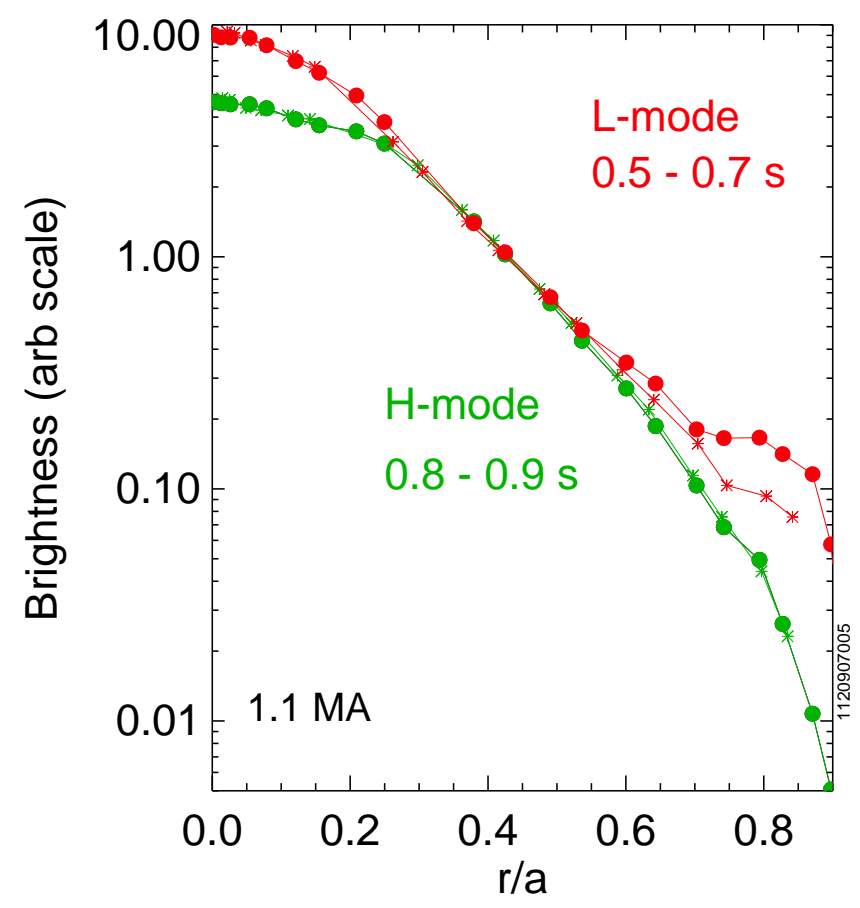

Figure 15: Bottom (dots) and top (asterisks) $\mathrm{Ar}^{17+} \mathrm{Ly}_{\alpha 1} \mathrm{x}$-ray brightness profiles for the 1.1 MA discharge of Fig.14 during the L-mode phase $0.5-0.7 \mathrm{~s}$ (red) and the Hmode phase $0.8-0.9 \mathrm{~s}$ (green). The $\mathrm{H}$-mode profiles have been reduced by a factor of 2.5 . 
mode profiles have been reduced by a factor of 2.5.) During the H-mode phase, there is no evidence for any up/down asymmetry, especially in the region $0.5<\mathrm{r} / \mathrm{a}<0.9$ where the feature appears in L- and I-mode. The edge brightness profiles in H-mode are very narrow compared to the L- and I-mode profiles since at these low electron temperatures, the upper $2 p$ levels can only be populated by radiative recombination of fully stripped $\mathrm{Ar}^{18+}$, but because of the strong inward impurity pinch in $\mathrm{H}$-mode (see Refs.[1-21] in [1]), there is insufficient $\mathrm{Ar}^{18+}$ (which must be born in the plasma core and diffused outwards quickly) at this radius. There is also a significant change to the background electron density profile in $\mathrm{H}$-mode for this discharge, as demonstrated in Fig. 16. During the L-mode phase, the profile has a modest gradient of $\sim 4.5 \times$

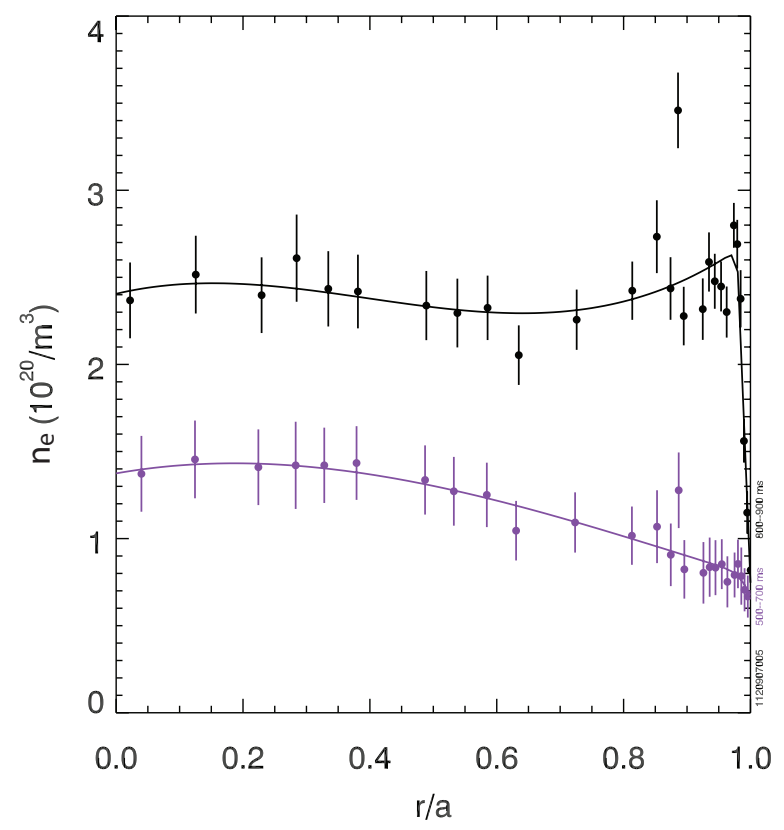

Figure 16: Electron density profiles during the L-mode (purple dots) and H-mode (black dots) phases of the discharge shown in Fig. 14.

$10^{20} / \mathrm{m}^{4}$ between $\mathrm{r} / \mathrm{a}=0.4$ and 0.9 . In contrast, during the $\mathrm{H}$-mode phase, there is a steep pedestal between $\mathrm{r} / \mathrm{a}=0.9$ and $1.0\left(\sim 6 \times 10^{22} / \mathrm{m}^{4}\right)$, while inside $\mathrm{r} / \mathrm{a}=0.9$ the profile is very flat (even slightly negative) with a very small gradient. These and other parameters are recorded in the last two rows of Table 1.

To summarize the findings of this section, the magnitude of the up/down impurity density asymmetry is seen to scale as $1 / \mathrm{I}_{\mathrm{P}} \mathrm{n}_{e} \sqrt{T_{e}}$ (that is, independently with each of 
these parameters) and become very small in $\mathrm{H}$-mode when the electron density gradient in the pedestal becomes very steep and in the core becomes flat.

\section{Discussion and Conclusions}

In order to understand the behavior documented in the previous section, it is informative to compare the results to the predictions of neo-classical theory. Certainly the direction of the asymmetry is in agreement $[11,40,12,16,41,42]$. The up/down impurity density is treated as an $m=1$ perturbation given as $\tilde{n} / \mathrm{n}$, which is equivalent to $($ ratio -1$) /($ ratio +1$)$, where 'ratio' denotes the up/down density ratio. Early treatments $[40,12]$ valid for small perturbations (with $\tilde{n} / \mathrm{n}<$ inverse aspect ratio) in circular plasmas found that the asymmetry is proportional to the product of

$$
2 \sqrt{2} \frac{q^{2} Z_{I}^{2}}{\tau_{i i} \omega_{c i}}
$$

and factors dependent on the normalized inverse density and/or temperature gradient scale lengths, depending on the collisionality regime. Here $\mathrm{q}$ is the safety factor, $\mathrm{Z}_{I}$ is the impurity charge, $\tau_{i i}$ is the collision time and $\omega_{c i}$ is the cyclotron frequency. In the Pfirsch-Schlüter regime, $\tilde{\mathrm{n}} / \mathrm{n} \sim \nabla \mathrm{n} / \mathrm{I}_{\mathrm{P}}{ }^{2} \mathrm{~T}^{3 / 2}$ at fixed magnetic field. This qualitatively captures some of the observed behavior, although measured dependences on current and temperature are not so strong, and there is no predicted relation with density. However, the observed magnitude is much larger than the region of validity for the calculation. Even in treatments valid for strong asymmetries [41, 42], the maximum $\tilde{\mathrm{n}} / \mathrm{n} \sim 0.3$, is much smaller than the observations, while the leading parameter dependence is the same as in Eq. (2). Furthermore, the detailed parameter scaling is not in agreement with observations, as demonstrated in Fig. 17, which shows the measured $\tilde{\mathrm{n}} / \mathrm{n}$ as a function of $\mathrm{q}^{2} \nabla \mathrm{n} / \mathrm{BT}^{3 / 2}$. It is difficult to see much correlation here except to say that the H-mode discharges with very small density gradients for r/a between 0.8 and 0.9 have very small impurity density asymmetries.

A more general global neo-classical treatment that includes radial transport may provide an explanation for the larger observed up/down impurity density asymmetry than suggested by [42], which treated the main ions in the local limit and retained scale separation between parallel and radial flows. Significant departures from local neoclassical results have been predicted in the edge region where gradient scale lengths become comparable to the poloidal gyroradius $[43,44]$. In standard local neo-classical theory relevant to the core, radial transport is much slower than parallel transport, so that densities, potentials, and temperatures remain nearly constant on the flux surfaces. However, in the edge region, radial transport due to either turbulence or collisional processes competes with parallel transport [43] and must be included at the same order in the continuity equation, modifying the main ion surface flows. The radial fluxes from turbulence tend to be largest on the outer midplane. The deuterium parallel and poloidal flows exhibit strong poloidal variation, as does the electrostatic potential [43]. The corresponding impurity parallel flows would then be modified by the strongly asymmetric 


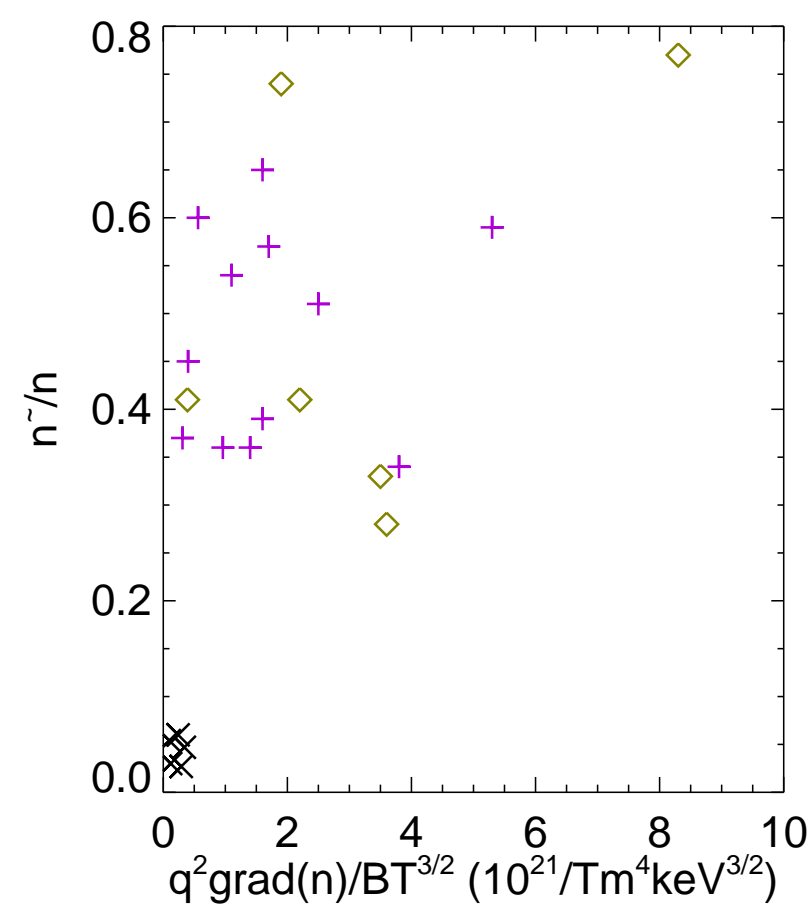

Figure 17: The measured impurity density asymmetry $\tilde{n} / \mathrm{n}$ as a function of the expression $\mathrm{q}^{2} \nabla \mathrm{n} / \mathrm{BT}^{3 / 2}$ evaluated at $\mathrm{r} / \mathrm{a}=0.85$ for selected discharges. Mustard diamonds, purple plus signs and black Xs denote Ohmic, I-mode and H-mode plasmas, respectively. 
friction forces from the main ions in proportion to $\mathrm{n}_{Z} \mathrm{Z}_{I}{ }^{2}$ [42], so that their flows amplify the main ion flow asymmetries. This impurity flow asymmetry, along with the main ion density and potential asymmetry, would result in strong impurity density asymmetries. The main ion finite orbit width spreads these asymmetries radially inward to the steep gradient edge region [43]. The role of radial transport, particularly due to turbulence, in this scenario would be qualitatively consistent with the observation that asymmetries are strong in L-mode and I-mode, but absent in H-mode. In addition, the directions of plasma current and collisionality variation would remain qualitatively consistent with observation. To treat this problem consistently in more collisional Alcator C-Mod plasmas where the ion temperature gradient scale length is comparable to the deuterium poloidal gyroradius, a full-f simulation may be needed. Recently [45], a full-f two fluid global edge turburbulence model has been applied to this problem, and up/down ion density and temperature asymmetries have been demonstrated.

There is evidence that the up/down impurity density asymmetry is driven by turbulent processes. Edge turbulence levels in L-mode plasmas have been found to be up/down asymmetric [46, 47] and to switch sides with a reversal of the toroidal magnetic field [46]. It has been pointed out that parallel impurity transport, which could give rise to an up/down asymmetry, is linked to cross-field transport [18] when parallel and perpendicular transport times are comparable. Indeed, in L- and I-mode, radial impurity transport has been shown to be highly anomalous $[48,1]$, with an effective cross-field diffusivity, $\mathrm{D}_{\text {eff }}$, of $0.2-0.5 \mathrm{~m}^{2} / \mathrm{s}$, which is significantly larger than neoclassical levels.

It is interesting to note that the measured impurity diffusivity in C-Mod L-mode plasmas decreases with plasma current as $\mathrm{D}_{\text {eff }} \propto \mathrm{I}_{\mathrm{P}}{ }^{-.7}$ [1]. Similar observations have been made on other devices $[49,50,51]$. At these outer radii with the electron temperature below $1 \mathrm{keV}$, the dominant population mechanism for the upper levels of the $\mathrm{Ar}^{17+} \mathrm{Ly}_{\alpha}$ doublet is radiative recombination of fully stripped $\mathrm{Ar}^{18+}$, which must be born in the plasma core and then transported radially outwards. Higher diffusivity at lower plasma current would lead to a larger abundance of $\mathrm{Ar}^{18+}$ at the outer locations. A similar decrease in the impurity diffusivity with plasma current in I-mode has also been documented [1].

From observations of the impurity confinement time at different ICRF power levels [1], it was found that $\mathrm{D}_{\text {eff }}$ increases with the core electron temperature. This would be in contrast to the expectation that the higher impurity diffusivity at higher temperature would lead to an abundance of the $\mathrm{Ar}^{18+}$ required for recombination. On the other hand, the radiative recombination rate increases as the electron temperature decreases, so this would favor higher population of the transition upper levels. It is not possible to explore this effect in Ohmic discharges without auxiliary heating power since the electron temperature is fixed at fixed density. The impurity confinement time $\tau_{I}$ is independent of density for Ohmic plasmas [1]; similar observations are seen in Imode. In H-mode plasmas, where the up/down asymmetry disappears, there is a large neo-classical-like edge inward pinch (see Refs.[1-21] in [1]). This prevents the fully stripped $\mathrm{Ar}^{18+}$ from reaching the plasma edge and the perpendicular transport time is much larger than the parallel transport time.

Hopefully this study has shed new light on this long-standing phenomenon observed in tokamak plasmas. 


\section{Acknowledgements}

The authors thank the Alcator C-Mod operations and ICRF groups for expert running of the tokamak. Work supported at MIT by DoE Contract No. DE-FC02-99ER54512 and at ORNL by DoE Contract No. DE-AC05-00OR22725. JMAA would like to thank Clare Hall, University of Cambridge for very helpful support with Fortran resource access for the COLRAD code runs.

\section{References}

[1] J.E. Rice et al., 2015 Nucl. Fusion 55033014.

[2] P. Smeulders et al., 1986 Nucl. Fusion 26267.

[3] H. Chen et al., 2000 Phys. Plasmas 74567.

[4] L.C. Ingesson et al., 2000 Plasma Phys. Control. Fusion 42161.

[5] K.D. Marr et al., 2010 Plasma Phys. Control. Fusion 52055010.

[6] M.L. Reinke, 2011 Experimental tests of parallel impurity transport theory in tokamak plasmas Ph.D. Thesis Massachusetts Institute of Technology

[7] M.L. Reinke et al., 2012 Plasma Phys. Control. Fusion 54045004.

[8] T. Pütterich et al., 2012 Nucl. Fusion 52083013.

[9] R.M. Churchill et al., 2013 Nucl. Fusion 53122002.

[10] C. Angioni et al., 2014 Nucl. Fusion 54083028.

[11] J.L. Terry et al., 1977 Phys. Rev. Lett. 391615.

[12] K. Brau et al., 1983 Nucl. Fusion 231657.

[13] K.W. Wenzel, 1990 Measurements of injected impurity transport in TEXT using multiply filtered soft x-ray detectors Ph.D. Thesis Massachusetts Institute of Technology

[14] R. Durst et al., 1992 Nucl. Fusion 322238.

[15] S.P. Regan, 1996 Soft x-ray spectroscopy on the Phaedrus-T tokamak Ph.D. Thesis Johns Hopkins University

[16] J.E. Rice et al., 1997 Nucl. Fusion 37241.

[17] J.E. Rice et al., 2013 Nucl. Fusion 53033004.

[18] M.L. Reinke et al., 2013 Nucl. Fusion 53043006.

[19] E.S. Marmar et al., 2007 Fusion Sci. Technol. 51261. 
[20] M. Greenwald et al., 2014 Phys. Plasmas 21110501.

[21] P.T. Bonoli et al., 2007 Fusion Sci. Technol. 51401.

[22] M. Greenwald et al., 1997 Nucl. Fusion 37793.

[23] D.G. Whyte et al., 2010 Nucl. Fusion 50105005.

[24] A.E. Hubbard et al., 2016 Nucl. Fusion 56086003.

[25] L.L. Lao et al., 1985 Nucl. Fusion 251611.

[26] J.W. Hughes et al., 2003 Rev. Sci. Instrum. 741667.

[27] N.P. Basse et al., 2007 Fusion Sci. Technol. 51476.

[28] R.M. McDermott et al., 2009 Phys. Plasmas 16056103.

[29] C. Theiler et al., 2014 Nucl. Fusion 54083017.

[30] R.M. Churchill et al., 2015 Phys. Plasmas 22056104.

[31] A. Ince-Cushman et al., 2008 Rev. Sci. Instrum. 79 10E302.

[32] M.L. Reinke et al., 2012 Rev. Sci. Instrum. 83113504.

[33] J.E. Rice et al., 2017 Nucl. Fusion 57116004.

[34] E.S. Marmar et al., 1986 Phys. Rev. A 33774.

[35] J.E. Rice et al., 2011 J. Phys. B 44165702.

[36] N.N. Ljepojevic, R.J. Hutcheon and J. Payne, 1987 Comput. Phys. Commun. 44 157.

[37] J.M.A. Ashbourn and N.N.Ljepojevic 1995, Phys. Rev. A 524966.

[38] J.M.A. Ashbourn 1999, Phys. Rev. E 596198.

[39] F.F. Goryayev et al, 2006 arXiv:physics/0603164v2 [physics.atom-ph]

[40] K.H. Burrell and S.K. Wong, 1979 Nucl. Fusion 191571.

[41] D.J.Sigmar et al., in Theory of Fusion Plasmas (Proc. Joint Varenna-Lausanne Int. Workshop, 1990), Editrice Compositori, Bologna (1990) 65.

[42] T. Fülöp and P. Helander, 2001 Phys. Plasmas 83305.

[43] M. Landreman and D.R. Ernst, 2012 Plasma Phys. Control. Fusion 54115006.

[44] M. Landreman and D.R. Ernst, 2013 J. Comp. Phys. 243130.

[45] B. Zhu et al., 'Up-down symmetry breaking in global tokamak edge simulations', 2018 submitted to Nucl. Fusion. 
[46] D. Brower et al., 1985 Phys. Rev. Lett. 54689.

[47] C. Fenzi et al., 1999 Plasma Phys. Control. Fusion 411043.

[48] J.E. Rice et al., 1997 Phys. Plasmas 41605.

[49] E.S. Marmar et al., 1980 Phys. Rev. Lett. 452025.

[50] E.S. Marmar et al., 1982 Nucl. Fusion 221567.

[51] M. Mattioli et al., 1995 Nucl. Fusion 351115. 